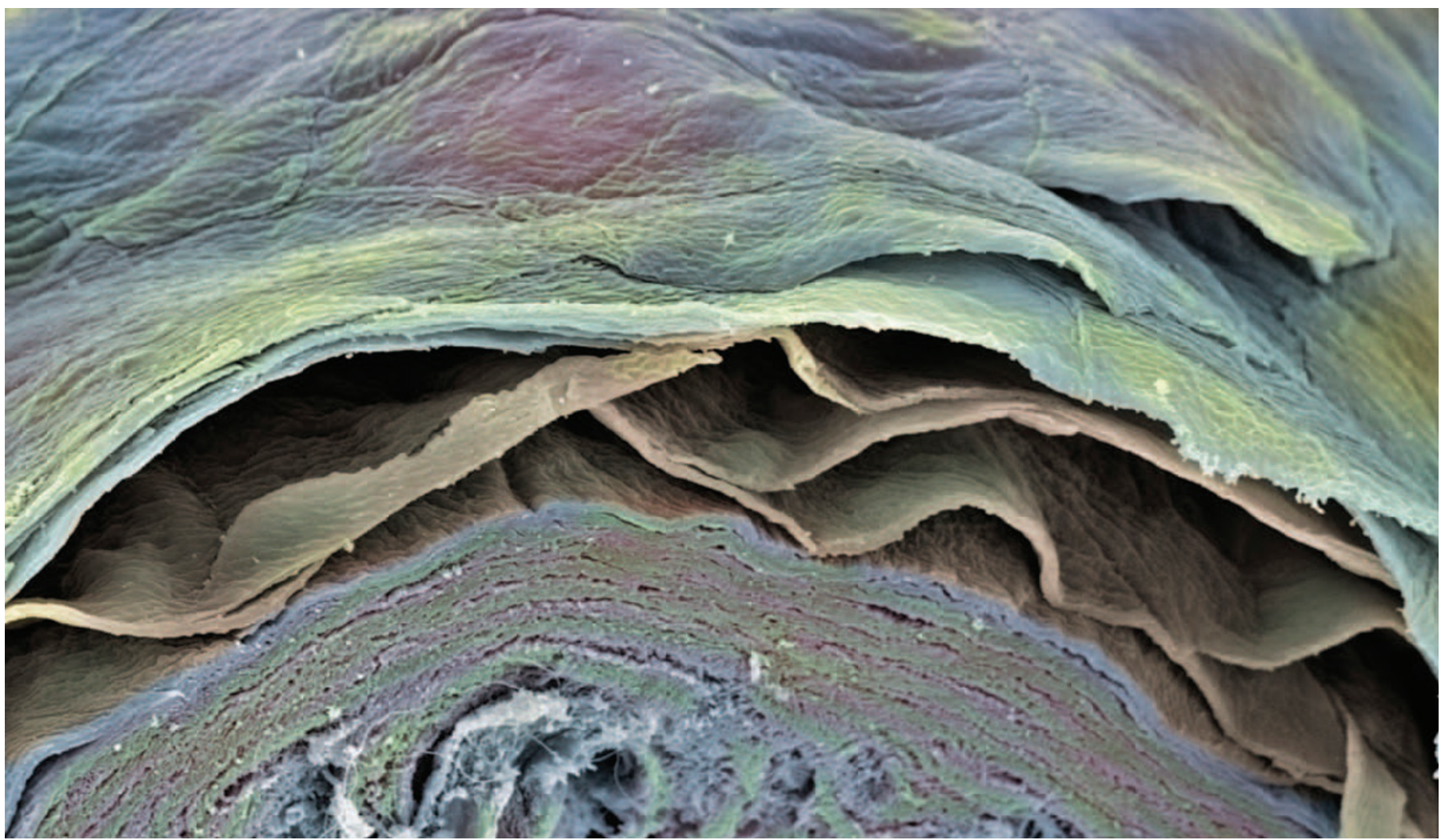

The orderly layers of healthy skin contrast with the eruptions of eczema (right image).

SKIN

\section{Into the breach}

\section{A focus on skin barrier disorders has opened up new thinking about how allergies kick in.}

\section{BY CLAIRE AINSWORTH}

$\mathrm{I}$ n the autumn of 2005, Alan Irvine, a dermatologist at Trinity College Dublin, noticed something unusual about a cohort of patients with ichthyosis vulgaris - a dry, scaly skin disease. Irvine had been working with geneticist Irwin McLean at University of Dundee, UK, whose team had recently identified two mutations in the gene behind the inherited disorder. But Irvine's new observation pointed to something much bigger: a radical new explanation of what causes allergic diseases.

Irvine had spotted that far more ichthyosis vulgaris patients also had atopic eczema compared to the general population. Could their skin be at fault? Indeed, could epithelium defects be responsible for other allergies too, such as asthma, allergic rhinitis and food allergies, rather than a misfiring adaptive immune system as was widely believed? While the two hypotheses are not mutually exclusive - faults in both could combine to cause allergies - the idea that the epithelium is a major player could explain several phenomena, including why people with atopic eczema often go on to get a set of other allergies. The epithelium is being targeted in new approaches to allergy treatments. Stephen Holgate, a clinician and asthma researcher at Southampton University, UK, describes it as "the new boy on the block".

\section{REVEALING MUTATIONS}

The conventional view of allergies is that some people have an inherited tendency to develop multiple allergies - a condition known as atopy - because of a faulty immune response, dominated by the activity of Th2 lymphocytes and a type of antibody called immunoglobulin E (IgE). A decade ago, however, hints started emerging that this wasn't the entire story.

One clue came from research into a rare skin disease called Netherton syndrome. Netherton sufferers have fragile, scaldedlooking skin prone to cracking, and they develop rampant atopy. Netherton syndrome

is a recessive monogenic condition, caused by mutations to the SPINK5 gene. The normal version of SPINK5 encodes a protein that inhibits the activity of certain skin proteases (enzymes that cut up proteins). These serine proteases are needed for everyday skin renewal, but if they become active too early, as happens with Netherton syndrome, they destroy the younger layers of skin before they mature. Bacteria and other organisms present in the environment also produce proteases, such as those found in pollen and in the droppings of that bane of asthma sufferers, the house dust mite, whose proteases Der $\mathrm{p}$ I and Der $\mathrm{p}$ II damage epithelia.

In 2001, a team at the Wellcome Trust Centre for Human Genetics in Oxford, UK, showed that a particular variant of SPINK5 was strongly associated with eczema and asthma in families with atopy, but who did not have Netherton syndrome. This suggested to the researchers that lesions in the epithelial barrier might be predisposing people to eczema, and that the atopy might be secondary.

Three years later came the breakthrough involving McLean's team in Dundee and Irvine's team in Dublin. Their work focused on the filaggrin

This theory shifts the blame from the adaptive immune response to the innate one. gene FLG, which is needed to help keratinocytes form and moisturise the skin's outer impermeable layer, the 


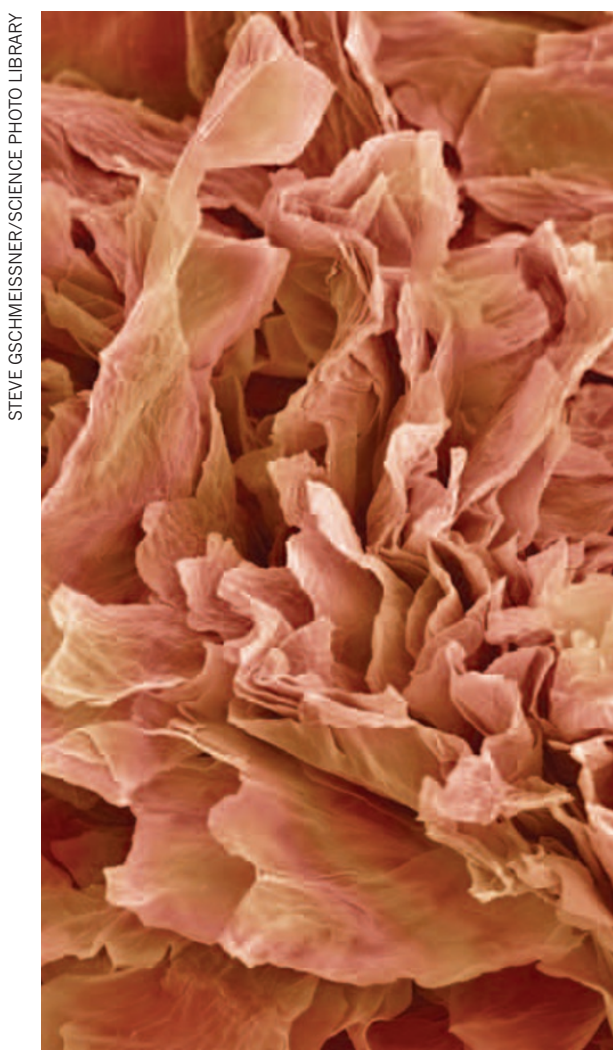

stratum corneum. Only about 1 in 100 northern Europeans has ichthyosis vulgaris resulting from mutations in the FLG gene, but 1 in 10 northern Europeans is heterozygous, carrying one mutated copy and one normal copy. Irvine noted that the heterozygous carriers, although free of full-blown ichthyosis, nonetheless had overly creased palms and unusually dry skin, suggesting that it was more fragile than normal. They compared 50 children with ichthyosis vulgaris with 200 unaffected individuals and found a remarkably strong association between FLG mutations and eczema.

\section{MORE THAN SKIN DEEP}

Subsequent work, says Irvine, showed that eczema patients who carry FLG mutations tend to have a more severe form of eczema, and are more disposed to develop a series of other allergies too, a phenomenon known as the 'atopic march'. The finding that filaggrin mutations are strongly associated with asthma was a particular surprise, since filaggrin is not expressed in the lung. Furthermore, McLean's dermatologist colleague Sara Brown, also at Dundee, found a strong association between filaggrin mutations and peanut allergy. Filaggrin has not been detected in the gut, but is found in the lining of the mouth as well as in the skin.

These and other findings, including the link between atopy and another rare fragile skin disorder called peeling skin syndrome, are consistent with the idea that allergies are triggered by a leaky epidermis. Although the precise mechanism is poorly understood, it is thought that leaky skin allows allergens to penetrate the body and prime the immune system for an allergic reaction. Recent work in a murine model tested the concept. Mice lacking filaggrin have dry, flaky skin that is abnormally thin and porous; if such mice have their skin smeared with ovalbumin, a protein abundant in egg white, they develop a systemic IgE allergic response to it, while normal mice do not.

"Dermatologists had always said they thought that eczema was primarily a skin disease, and that the effects throughout the body were secondary to that," says Brown. In the past few years, the field has exploded, extending the faulty epithelium idea to allergic rhinitis (porous nasal epithelium) and food allergies (leaky gut epithelium).

The intriguing thing about this theory is that it shifts the blame from the adaptive immune response to the innate one. Epidermal cells, long thought to form only a passive barrier to invaders, are now emerging as players. Keratinocytes not only present antigens to immune cells, they also secrete cytokines such as thymic stromal lymphopoietin (TSLP), which drives atopic march (see 'Marching with allergies', page S14) as well as other cytokines that fan the Th2dominated immune response. "The epidermis is quite immunologically active," says Irvine. "It isn't just inert bricks and mortar."

\section{ASTHMATIC COMPLICATIONS}

Meanwhile, asthma researchers are questioning the idea that the adaptive immune system is the sole trigger of allergy. Over the past 20 years or so, a growing body of work has shown that the airway epithelium seems to be more fragile in asthma patients. For example, Holgate's team in Southampton has shown that tight junctions, the massive protein complexes that help hold cells together in epithelia, do not assemble properly in the airways of asthmatic patients. Other teams have found that faults in different protein complexes also involved in epithelial cell junctions are linked to asthma and that the airway epithelium in asthmatics is poorly repaired following injury.

What's more, studies of cultured lung epithelia show that several common triggers of asthma also disrupt cell junctions, particularly in epithelia from asthmatic patients. And some allergens compound their effect by stimulating the immune system. The house dust mite protease allergen Der p II, for instance, interacts with innate immune cell receptors called Toll-like receptors, triggering a 'danger signal' that attracts the attention of the adaptive immune response. "If you add that to the epithelial weakness," says Holgate, "you have a perfect storm." -

Claire Ainsworth is a freelance science writer based in Southampton, UK.
SCOPE FOR TREATMENT Fixing the barriers

Academic researchers and pharmaceutical companies are already investigating therapies that bolster the integrity of epithelia as a treatment for allergies.

For example, Irwin McLean and colleagues at University of Dundee, UK, are searching for small molecules that could boost the production of filaggrin and perhaps other components of the skin barrier machinery in eczema patients. They are also investigating drugs that could help epithelial cells 'ignore' so-called nonsense mutations to the FLG gene - nonsense mutations are the most common mutations in the filaggrin gene in northern Europeans, and cause the cell's protein synthesis machinery to halt part way through translating the genetic code into the protein.

Certain kinds of antibiotics called aminoglycosides are already known to let cells read-through nonsense mutations, and drugs based on the read-though approach are in clinical trials for other genetic diseases such as cystic fibrosis.

McLean and his colleagues have been awarded a patent covering the use of readthrough drugs, including aminoglycosides such as the off-patent antibiotic gentamicin, for the treatment of ichthyosis vulgaris and other atopic conditions. Such drugs would enable cells to bypass nonsense mutations and thereby restore filaggrin production. The treatment could help the skin develop a more natural, stronger structure. Gentamicin, however, has harmful side effects, so the team is screening for alternative read-though drugs and is following a number of leads in cell and animal models.

Another way of strengthening the epithelium is to help it repair itself. The airway epithelium of asthma patients is known to be poor at self-repair, but experiments in tissue culture suggest that its ability to regenerate improves in response to growth factors. Holgate's team at Southampton University, UK, is currently testing the safety and efficacy of keratinocyte growth factor (KGF) on asthma patients. KGF is present in the skin and the lining of the gut, where it promotes the growth of cells that help repair damage and maintain tissue strength. Work by other researchers in Lille and Paris,

France, on a rat model of asthma suggests that KGF can reduce inflammation and leakiness in the airway epithelium, as well as making it more resistant to being damaged by allergens. 